\title{
The Association between Body Mass Index and Craniometrical Parameters in Slovak Population (Original paper)
}

\section{A. Nadazdyova (Anna Nadazdyova)', E. Stefankova (Eva Stefankova)², M. Samohyl (Martin Samohyl) ${ }^{3}$}

${ }^{1}$ Department of Stomatology and Maxilofacial Surgery,

Faculty of Medicine, Comenius University in Bratislava, Slovakia

2 Department of Pediatric Surgery, Children teaching hospital,

Faculty of Medicine, Comenius University in Bratislava, Slovakia

${ }^{3}$ Institute of Hygiene, Faculty of Medicine, Comenius University in Bratislava, Slovakia

\section{E-mail address:}

martin.samohyl@fmed.uniba.sk

\section{Reprint address:}

Martin Samohyl

Comenius University in Bratislava

School of medicine

Bratislava, Slovakia

Suource: Clinical Social Work and Health Intervention Pages: $28-30$
Volume: 8

Issue: 3

\section{Reviewers:}

George Benca

House of family St. Max Kolbe, Children's Hospital Clinic Phnom Penh

Daria Kimuli

St. Kizito centre Nairobi, Kenya

\section{Key words:}

Craniometrical Parameters, Body Mass Index, Slovak Population.

\section{Publisher:}

International Society of Applied Preventive Medicine i-gap

CSWHI 2017; 8(3): 28 - 30; DOI 10.22359/cswhi_8_3_05 @ 2017 Clinical Social Work and Health Intervention

\section{Abstract:}

Anthropometry can detect shape changes over time. This is important to diagnose acquired malformations. The term surface anthropometry is 
used in this paper to refer to the measurement of the facial surface features (1). One of the major reasons patients seek orthodontic treatment is to improve their facial appearance (2). Currently, two non-invasive methods can be used to collect quantitative soft tissue facial data in three dimensions: direct; conventional anthropometry; digital/computerized anthropometry. Body mass index except of important impact to the craniofacial parameters also has a significant influence on e.g. the blood pressure (cardiovascular risk) $(3,4)$.

The aim of this study is to find any association between the mean values of craniofacial and BMI.

\section{The study}

The sample consisted of 100 patients (50.0\% men, 50.0\% women) aged between 18-32 years (mean age $23.09 \pm 2.70$ years) attending dental surgeries in Bratislava (2013 - 2016). In this paper these craniofacial parameters were analyzed: nose breadth, bi-entocanthion breadth, bi-zygomatic breadth, bi-gonial breadth, total facial height, mouth breadth, morphologic face height, upper-lip height, lower-lip height and pupils - mid-face (right). The analyzed group of patients included measurements provided by directed anthropometry

Table 1: Mean, minimum and maximal values of craniofacial parameters according to BMI $(n=100)$

\begin{tabular}{|c|c|c|c|c|c|c|c|c|}
\hline \multirow{2}{*}{$\begin{array}{l}\text { Craniofacial } \\
\text { Parameters }\end{array}$} & \multicolumn{3}{|l|}{ Study Group } & \multirow{2}{*}{\begin{tabular}{|l|} 
Mean \\
$\mathbf{x}$ (SD)
\end{tabular}} & \multirow{2}{*}{$\begin{array}{l}\text { Medi- } \\
\text { an }\end{array}$} & \multirow[t]{2}{*}{ Min } & \multirow[t]{2}{*}{ Max } & \multirow[t]{2}{*}{$\mathbf{p}$} \\
\hline & & & $\mathbf{n}$ & & & & & \\
\hline \multirow{2}{*}{$\begin{array}{l}\text { al-al }(\mathrm{cm}) \\
\text { nose breadth }\end{array}$} & \multirow{2}{*}{ BMI $\left[\mathrm{kg} \cdot \mathrm{m}^{-2}\right]$} & 18.6-24.9 & 83 & $3.45(0.36)$ & 3.40 & 2.64 & 4.60 & \multirow{2}{*}{0.018} \\
\hline & & $>25.0$ & 14 & $3.70(0.33)$ & 3.70 & 3.20 & 4.28 & \\
\hline \multirow{2}{*}{$\begin{array}{l}\text { en-en }(\mathrm{cm}) \\
\text { bi-entocanthion } \\
\text { breadth }\end{array}$} & \multirow[b]{2}{*}{ BMI $\left[\mathrm{kg} \cdot \mathrm{m}^{-2}\right]$} & 18.6-24.9 & 83 & $2.98(0.38)$ & 2.90 & 2.30 & 4.00 & \multirow[b]{2}{*}{0.432} \\
\hline & & $>25.0$ & 14 & $3.06(0.34)$ & 3.17 & 2.37 & 3.60 & \\
\hline \multirow{2}{*}{$\begin{array}{l}\text { zy-zy }(\mathrm{cm}) \\
\text { bi-zygomatic breadth }\end{array}$} & \multirow{2}{*}{ BMI $\left[\mathrm{kg} \cdot \mathrm{m}^{-2}\right]$} & $18.6-24.9$ & 83 & $12.54(0.99)$ & 12.60 & 10.50 & 14.30 & \multirow{2}{*}{0.03} \\
\hline & & $>25.0$ & 14 & $13.33(1.19)$ & 13.05 & 11.02 & 15.80 & \\
\hline \multirow{2}{*}{$\begin{array}{l}\text { go-go }(\mathrm{cm}) \\
\text { bi-gonial breadth }\end{array}$} & \multirow{2}{*}{ BMI $\left[\mathrm{kg} \cdot \mathrm{m}^{-2}\right]$} & 18.6-24.9 & 83 & $11.22(0.95)$ & 10.90 & 10.00 & 13.80 & \multirow{2}{*}{0.489} \\
\hline & & $>25.0$ & 14 & $11.50(1.42)$ & 10.55 & 10.00 & 13.80 & \\
\hline \multirow{2}{*}{$\begin{array}{l}\text { n-gn }(\mathrm{cm}) \\
\text { total facial height }\end{array}$} & \multirow{2}{*}{$\mathrm{BMI}\left[\mathrm{kg} \cdot \mathrm{m}^{-2}\right]$} & 18.6-24.9 & 83 & $11.71(0.89)$ & 11.80 & 9.50 & 13.70 & \multirow{2}{*}{0.001} \\
\hline & & $>25.0$ & 14 & $12.55(0.73)$ & 12.80 & 11.50 & 13.70 & \\
\hline \multirow{2}{*}{$\begin{array}{l}\text { ch-ch }(\mathrm{cm}) \\
\text { mouth breadth }\end{array}$} & \multirow{2}{*}{$\mathrm{BMI}\left[\mathrm{kg} \cdot \mathrm{m}^{-2}\right]$} & 18.6-24.9 & 83 & $5.04(0.45)$ & 5.00 & 3.41 & 6.00 & \multirow{2}{*}{0.001} \\
\hline & & $>25.0$ & 14 & $5.42(0.30)$ & 5.36 & 4.75 & 5.92 & \\
\hline \multirow{2}{*}{$\begin{array}{l}\text { sn-gn }(\mathrm{cm}) \\
\text { morphologic face } \\
\text { height }\end{array}$} & \multirow[b]{2}{*}{ BMI $\left[\mathrm{kg} \cdot \mathrm{m}^{-2}\right]$} & 18.6-24.9 & 83 & $6.32(0.71)$ & 6.40 & 4.60 & 8.30 & \multirow[b]{2}{*}{0.041} \\
\hline & & $>25.0$ & 14 & $6.79(0.74)$ & 6.74 & 5.50 & 8.31 & \\
\hline \multirow{2}{*}{$\begin{array}{l}\text { Ls-Stm }(\mathrm{cm}) \\
\text { upper-lip height }\end{array}$} & \multirow{2}{*}{$\mathrm{BMI}\left[\mathrm{kg} \cdot \mathrm{m}^{-2}\right]$} & $18.6-24.9$ & 83 & $0.70(0.22)$ & 0.70 & 0.10 & 1.20 & \multirow{2}{*}{0.495} \\
\hline & & $>25.0$ & 14 & $0.64(0.28)$ & 0.59 & 0.20 & 1.02 & \\
\hline \multirow{2}{*}{$\begin{array}{l}\text { Stm-Li }(\mathrm{cm}) \\
\text { lower-lip height }\end{array}$} & \multirow{2}{*}{ BMI $\left[\mathrm{kg} \cdot \mathrm{m}^{-2}\right]$} & $18.6-24.9$ & 83 & $1.04(0.17)$ & 1.03 & 0.56 & 1.50 & \multirow{2}{*}{0.588} \\
\hline & & $>25.0$ & 14 & $1.06(0.11)$ & 1.05 & 0.81 & 1.22 & \\
\hline Pupils- mid face & $\mathrm{BMI}$ & $18.6-24.9$ & 83 & $3.41(0.30)$ & 3.46 & 2.50 & 3.94 & 0884 \\
\hline$($ right $)(\mathrm{cm})$ & BMI [kg.m-2] & $>25.0$ & 14 & $3.42(0.30)$ & 3.50 & 2.80 & 3.70 & 0.884 \\
\hline
\end{tabular}


(PDAA) and from 3D scan (P3DAS). We have expected that participants with a BMI $>25$ will have a higher amount of fat in the face than participants with a BMI 18.6-24.9. The data were analyzed by the statistical program SPSS.

Mean values of craniofacial parameters according to BMI are presented in Table 1. The differences between BMI 18.6-24.9 and $\mathrm{BMI}>25.0$ had significant effect on the evaluation of nose breadth $(3.45 \pm 0.36 \mathrm{~cm}$ vs. $3.70 \pm 0.33 \mathrm{~cm} ; \mathrm{P}=0.018)$, bi-zygomatic breadth $(12.54 \pm 0.99 \mathrm{~cm}$ vs. $13.33 \pm 1.19 \mathrm{~cm}$; $\mathrm{P}=0.031)$, total facial height $(11.71 \pm 0.89 \mathrm{~cm}$ vs. $12.55 \pm 0.73 \mathrm{~cm} ; \mathrm{P}=0.001)$, mouth breadth $(5.04 \pm 0.45 \mathrm{~cm}$ vs. $5.42 \pm 0.30 \mathrm{~cm} ; \mathrm{P}=0.001)$ and morphologic face height $(6.32 \pm 0.71 \mathrm{vs}$. $6.79 \pm 0.74 \mathrm{~cm} ; \mathrm{P}=0.041$ ).

In conclusion BMI $>25.0$ had significant impact on high proportions of facial tissue than BMI 18.6-24.9 in this parameter: upper-lip height in relationship with these parameters; the nose breadth, bi-zygomatic breadth, total facial height, breadth and mouth morphologic face height.

\section{References}

1. FOURIE Z, DAMSTRA J, GERRITS PO et al (2011) Accuracy and repeatability of anthropometric facial measurements using cone beam computed tomography. Cleft Palate Craniofac J, 2011; 48 (5): 623-630.

2. DIMITRIOS K (2012) The impact of extraction vs nonextraction treatment on soft tissue changes in Class I borderline malocclusions. The Angle Orthodontist, 2012; 82 (2): 209-217.

3. JURKOVICOVA J, STEFANIKOVA Z, ARGALASOVA L (2009) Risk factors of cardiovascular diseases in the group of 65+ year olds of SR. Cardiology (2009) 18 (2): 47-62.

4. JURKOVICOVA J, STEFANIKOVA Z, ARGALASOVA L (2011) Selected life-style factors and nutrition habits associated with cardiovascular risk. Eur. J. Cardiovasc. Prev. Rehabil. 2011; 18 (Suppl. 1): S74. 